\title{
Outbursts from evolved massive stars: SN 2015bh and its relatives
}

\author{
Christina C. Thöne ${ }^{1}$, Antonio de Ugarte Postigo ${ }^{1,2}$ and Jose Groh ${ }^{3}$ \\ ${ }^{1}$ IAA-CSIC, Glorieta de la Astronomía s/n, 18008 Granada, Spain \\ email: cthoene@iaa.es \\ ${ }^{2}$ Dark Cosmology Centre, Niels-Bohr-Institute, Univ. of Copenhagen, Juliane Maries Vej 30, \\ 2100 Copenhagen, Denmark \\ ${ }^{3}$ Trinity College Dublin, The University if Dublin, Dublin 2, Ireland
}

\begin{abstract}
In their final stages, massive stars can show large eruptions which can resemble core-collapse IIn SNe. Here we present SN 2015bh in NGC 2770, a IIn/impostor, where archival data show variabilities for at least 21 years before the main event in 2015. Serendipitous spectra during an outburst are the only SN progenitor spectra available since SN 1987A and show an LBV with a fast, dense outflow. Analogues to SN 2015bh are SN 2009ip and SNhunt 248 while the SN 2000ch impostor could be equivalent to the outburst phase of SN 2015bh. It is currently unclear whether SN 2015bh (and SN 2009ip) were final core-collapse events. Alternatively, they might be large outbursts shedding the outer envelope and creating a Wolf-Rayet star in only a matter of decades. Future large-scale high-cadence surveys such as LSST will detect many more of these events and allow us a unique insight into the largely unknown late stages of massive stellar evolution.
\end{abstract}

Keywords. supernovae: individual (SN 2015bh, SN 2009ip), stars: evolution, stars: mass loss

\section{Introduction}

Luminous eruptions of massive stars are frequent, especially in the late stages of their evolution, but the mechanisms and processes are still poorly understood by theoretical models. So-called "SN impostors" are such massive outbursts that can reach almost the luminosity of a core-collapse SN, however, the event does not destroy the star. Eta Carinae, a luminous blue variable (LBV), is one of the most prominent examples with large documented outbursts in 1843 and 1892. Recently it had been suggested that it has experienced a major eruption once every about 300 years (Kiminki et al. 2016).

Another category of major outbursts are so-called "precursors" to SN events, which seem to be common in IIn SNe in the year prior to explosion (Ofek et al. 2014). The most typical case is an outburst with peaks several tens of days before the actual explosion and a luminosity several magnitudes below the final SN. In fact, they are so frequent that some relate this precursor to the actual SN and the later, brighter peak to the interaction with the circumstellar medium (Ofek et al. 2014). Possible precursors have also been observed in a SN IIb (Strotjohann et al. 2015) and in a Ic SNe (Corsi et al. 2014), although here they seem to be the exception. SNe IIb show some indication of extended envelopes or CSM interaction (indeed, the spectral evolution of IIb SNe change from pronounced hydrogen lines to He-dominated, Ib-like spectra and some of them show double-peaked lightcurves). The progenitors of SN IIn, IIb and Ic span a range of massive stars including LBVs, Wolf-Rayet (WR) stars and cool supergiants but all showing indications for different kinds of pre-explosion mass losses. Likewise, early observations of some SNe, so-called 
"flash-spectroscopy-SNe" (Gal-Yam et al. 2014), show narrow hydrogen lines from a dense CSM or envelope that disappear within days.

Here we present observations of the peculiar event SN 2015bh in NGC 2770 at a distance of $\sim 27 \mathrm{Mpc}$ which has hosted 3 other SNe prior to SN 2015bh (Thöne et al. (2017), see also Elias-Rosa et al. 2016). Archival observations over 21 years before the main event show variability of $\sim 2$ mag for two decades before a precursor with a peak in February 2015 and the brightest, so-called "main event" in May 2015. Our observations span the entire event from the precursor up to 200 days past maximum of the main event using the telescopes at Observatorio de Sierra Nevada (OSN), the Gran Telescopio Canarias (GTC) and Swift (Thöne et al. 2017). SN 2015bh shows many similarities to another famous event, SN 2009ip which also has several smaller outbursts documented prior to its possible explosion as IIn SN in 2012 (Pastorello et al. 2013, Mauerhan et al. 2013).

\section{SN 2015bh from 1994 to 2015}

Our search for the progenitor of SN 2015bh in archival data of NGC 2770 revealed that the star had been present in data at least back until 1994 and varying by about $\sim 2$ magnitudes over the years (Thöne et al. 2017). The progenitor was first detected during an outburst in 2013 by iPTF and named it PTF13efv, but it was not reported until the detection of the precursor in 2015 (Duggan et al. 2015). We obtained serendipitous imaging and spectra in Nov. 2013 while observing NGC 2770 and its satellite NGC 2770B just at the onset of the outburst later detected by iPTF, but did not realize the detection of an outburst until much later.

On Feb. 7, 2015, SNhunt reported a new transient later characterized as SN impostor or possible IIn (Elias-Rosa et al. 2015). This event was later known to be the precursor of SN 2015bh with a maximum $\sim 40$ days before the main event and a peak magnitude of $\mathrm{R}=-13.4$ mag. We initiated a follow-up campaign with the 0.9 and $1.5 \mathrm{~m}$ OSN telescopes which then lead to the discovery of a sudden rebrightening on May 16, 2015, the onset of the main event, which eventually reached an absolute magnitude of -17.5 mag around May 24. During the first month of the main event we obtained almost daily imaging and spectroscopy with OSIRIS at the GTC and Swift in UV and X-rays. Neither X-ray nor radio emission was detected from the SN and/or its progenitor (Thöne et al. 2017). By September 2015, the light curve had dropped to $\sim-12.5 \mathrm{mag}$ with a very shallow subsequent decay, implying that the material was not any more accelerated at this time. The light curve of the entire 21 years of observations is shown in Fig. 1.

The serendipitous spectra from the outburst in 2013 shows a typical LBV spectrum with a single P-Cygni profile and Balmer and Fe-lines, but no He-lines (this spectrum will be further discussed below). At the precursor in early 2015, a second absorption component emerges with a velocity of $\sim-2000 \mathrm{~km} \mathrm{~s}^{-1}$ in addition to the absorption at $-700 \mathrm{~km} \mathrm{~s}^{-1}$ already present in the LBV phase, suggesting a connection of the ejected material with the event that caused the precursor. At the onset of the main event, weak He lines furthermore start to appear. Around maximum of the main event the absorption components of the P-Cygni profile largely disappear but then later reemerge with a clear double P-Cygni profile in all lines while the He-lines had disappeared again. After the sun gap, the spectra have changed to broad nebular lines and the second, faster absorption now shows up as emission line accompanied by a small bump at the same velocity, but redshifted, suggesting some asymmetry in the ejecta. Our toy model for the explosion assumes a dense shell expelled some years before the main event which we see as the absorption component at $-700 \mathrm{~km} \mathrm{~s}^{-1}$. The faster material is expelled 


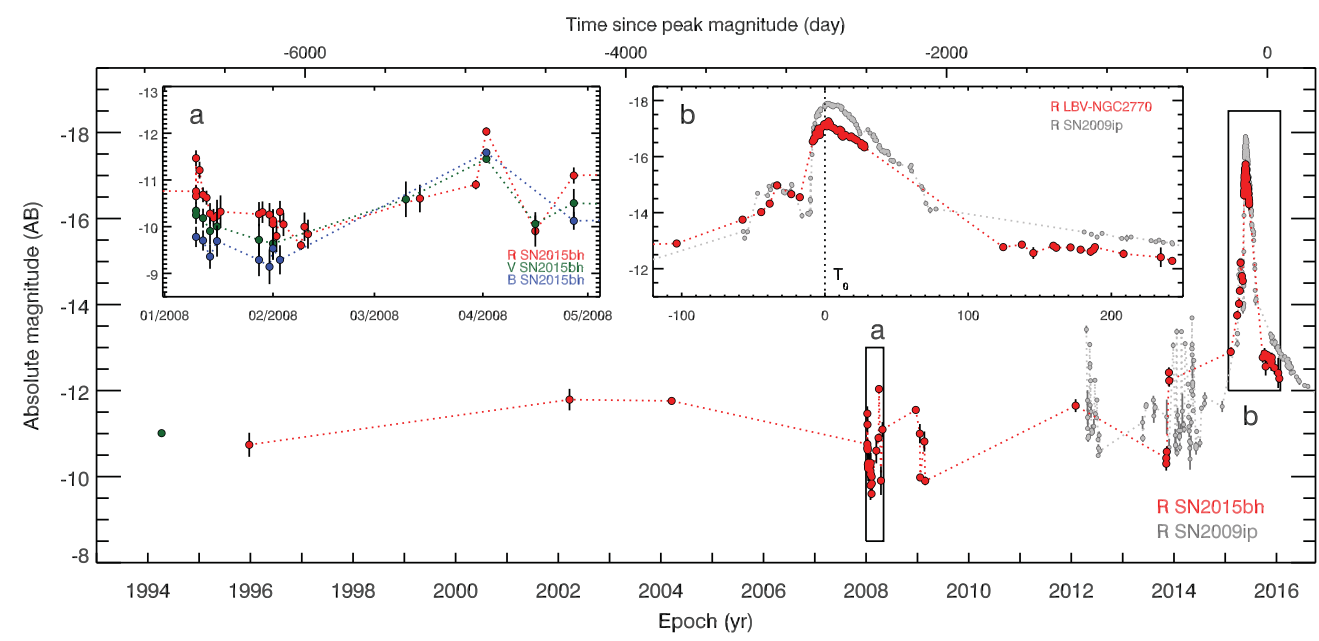

Figure 1. Lightcurve of SN 2015bh including the main event and precursor and the short term variations of its progenitor over 21 years. Inset a shows the dense monitoring of the progenitor during observations of SN 2008D and SN 2007uy in the same galaxy, inset b shows a blow-up of the precursor and main event. The grey dots are observations of the very similar event SN 2009ip shifted to the same $\mathrm{T}_{0}$ as $\mathrm{SN}$ 2015bh (from Thöne et al. 2017)

during the precursor and interacts with the dense wind of the progenitor giving rise to the luminosity of the main event. Some time around 100 days post explosion it catches up with the first larger shell and shows up in emission.

The main event had a total energy release of $1.2-1.8 \times 10^{48} \mathrm{erg}$. We model the SED during the main event with a simple BB model and derive the temperature and radius evolution. Until about 20 days past maximum the BB has an expansion velocity of $\sim 2200$ $\mathrm{km} \mathrm{s}^{-1}$ and a cooling rate of $660 \mathrm{~K} /$ day after which it slows down to $880 \mathrm{~km} \mathrm{~s}^{-1}$ and 150 $\mathrm{K} /$ day. The $\mathrm{H} \alpha \mathrm{EW}$ behaves very similar to those of other IIn SNe with a drop around maximum below $100 \AA$ and a subsequent rise to very high values of $>3000 \AA$ which could be indicative of a true core-collapse (Smith et al. 2014). The Balmer decrement $(\mathrm{H} \alpha / \mathrm{H}$ $\beta$ ) drops below the value for case B recombination (2.76) around maximum which could be explained by a very dense plasma thermalizing the emission. During the "nebular phase" it rises to $>10$, which is also seen in the nebular phase of other types of events. An explanation by increasing extinction from the ejecta is excluded as the SED shows no signs of reddening and dust production.

\section{The host galaxy NGC 2770 and the SN environment}

NGC 2770 was observed with different 3D techniques to study the ISM in this "SN factory" as well as the surroundings of the SNe. However, only our drift-scan spectroscopy run to observe the satellite galaxy of NGC 2770 happened to also cover the centre of the galaxy and the site of the progenitor, which is also the dataset from which we obtained the serendipitous spectra of the SN 2015bh progenitor at the onset of the 2013 outburst. As the spectra are contaminated by the progenitor emission we can only study the neighboring HII regions. The star is located in one of the outer spiral arms of NGC 2770 and in the same arm as SN 2007uy. This is different from SN 2009ip which was found at a large distance from its host. The regions near the SN site have a metallicity of around half solar and a stellar population age of 7-10 Myr, consistent with single star LBV models. 


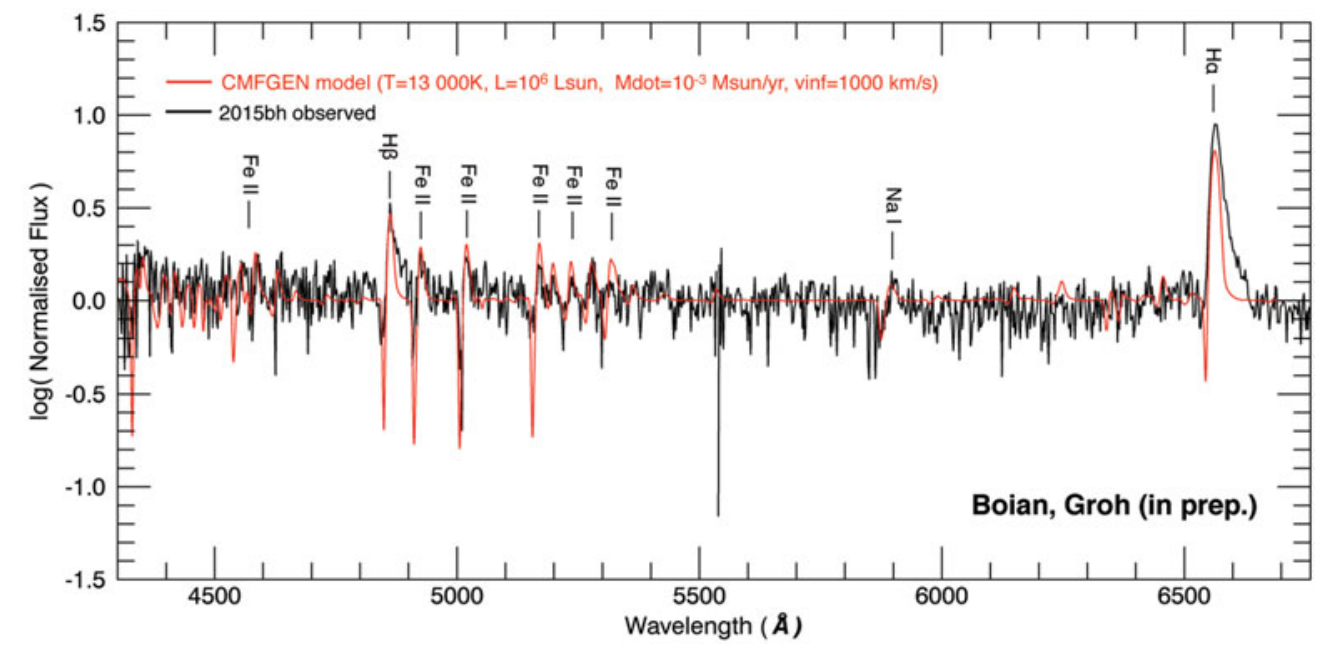

Figure 2. Modeling of the progenitor spectrum of SN 2015 at $-550 \mathrm{~d}$ with an LBV showing a dense, fast outflow using the radiation transfer code CMFGEN Hillier \& Miller 1998 The original spectrum is shown in black, the model spectrum in red.

Despite its elevated number of SNe, NGC 2770 does not show a particularly large starformation rate, the only remarkable fact is a large HI mass (Thöne et al. 2009). Likewise, there are little indications for a major merger event triggering large star formation (Thöne et al. in prep.). The HI mass could be indicative of an inflow of gas onto the galaxy, giving rise to a recent enhancement in star-formation and hence SN occurrence.

\section{The progenitor of SN 2015bh and its possible survivor}

Our serendipitous spectra from one of the outbursts in Nov. 2013 are probably the only available spectra of a SN progenitor prior to explosion since SN 1987A. We model the spectrum at $-550 \mathrm{~d}$ with template spectra of massive stars and find the best match with a luminous $\mathrm{LBV}\left(\mathrm{L}=10^{6} \mathrm{~L}_{\odot}\right)$ with a temperature of $13,000 \mathrm{~K}$ showing a dense, fast outflow of $\dot{M}=10^{-3} \mathrm{M}_{\odot}$ and $\mathrm{v}=1000 \mathrm{~km} \mathrm{~s}^{-1}$ (see Fig. 2). The large velocities indicate that the star is in outburst as this is too high for a usual stellar wind. Yellow hyper giant and WR models can be excluded with this model. Our model has a metallicity of about half solar, consistent with the metallicity of neighboring stellar populations in the host galaxy (see Sect. 3). Further details on this model will be given in Boian et al. in prep.

The progenitor of SN 2009ip has also been suggested to be a LBV star. Both SN 2015bh and SN 2009ip progenitors were always located red of typical LBVs in S-Dor outbursts at similar temperatures as Eta Carinae during its great eruption but at lower luminosities. During the pre-explosion event, the temperature stayed at similar values while the luminosity increased. The colors during the SN were typical of other IIn SNe. At late times, both SN 2009ip and SN 2015bh show a turn towards higher temperatures whose origin is still unclear. SN 2009ip now seems to be below the level of the proposed progenitor as observed in 1999 (Foley et al. 2011). At this point we cannot determine whether any of the two stars actually did explode. If they survived these outbursts, the stars might have lost most of their outer envelope and settle at a lower luminosity, while it seems they also turns towards higher temperatures similar to WR stars. LBVs have been suggested to be only a transitionary period on the evolution to a WR star (Maeder \& Meynet 2000), but lately LBVs have also been observed to directly explode 
as core-collapse SNe (Gal-Yam \& Leonard 2009). So far, it has been unclear how the star can loose enough mass in short time to evolve from a LBV to a WR star (Smith 2014). This kind of event might be the solution to this problem, expelling enough mass to transform a LBV in very short time into a WR star. In fact, many WR stars have been found to be embedded in SNR like shells. Another possibility is that these events happen in a binary system of which one star experiences core-collapse and the other, blue, companion emerges at late times. Late observations of the possible survivors will be crucial to confirm this possible scenario.

\section{A new type of event?}

SN 2015bh and its "twin" SN 2009ip are certainly not the only events of their type, in fact, more and more evidence appears that (very) massive stars at their late evolution stages show frequent, sometimes, massive outbursts. SN 2009ip also showed a complex absorption profile in the observed absorption lines indicating massive pre-explosion massloss (Margutti et al. 2014). Several other events have been suggested to be very similar to SN 2009ip (and hence SN 2015bh) such as SN 2010mc and LSQ13zm (see e.g. Elias-Rosa et al. 2016), however, most of them lack the extensive datasets of 2015bh and 2009ip, in particular at timescales long before the main event.

Two other interesting events in this context are the SNhunt 248 and SN 2000ch. The former has been largely neglected as possible member of this group, but shows a large number of similarities though at an overall lower luminosity: Variations of $\sim 2$ magnitude before the main event have been observed. The light-curve of the main event has been described as "triple-peaked". The first of these peaks corresponds to the precursor, the second is the peak of the main event. The third peak around $+100 \mathrm{~d}$ could be a late rebrightening indicating a further interaction with the CSM. This late peak, albeit with a lower increase in luminosity has in fact also been observed in SN 2009ip and if it occured for SN 2015bh at a similar time post maximum, it would have been missed as the SN had been in the sun gap at that time. SNhunt 248 has less and weaker pronounced absorption components than SN 2009ip and SN 2015bh but shares some similarities (see Kankare et al. 2015). SN 2000ch has been detected in 2000 as impostor and showed new outbursts in 2009-2010 (Pastorello et al. 2010) at similar magnitude variations and timescales as SN 2015bh. Also the spectra resemble those of SN 2015bh during the outburst in 2013. SN 2000ch might turn into a similar event than 2015bh at some point in the (near?) future and should certainly be monitored.

Detecting these events and other variations of massive stars prior to their SN explosion is still difficult and requires high-cadency, large-scale surveys. PTF and iPTF have discovered several candidate precursors, but most lie at the very limit of its observing capabilities. Obtaining spectra at time before the explosion is even more complicated and has so far only been obtained for SN 2015bh and SN 2000ch. Future deep all-sky survey such as LSST will hopefully detect many more of these outbursts prior to explosion and allow for more thorough follow-up. This will be very important to gain insight in the so-far poorly understood late stages of massive stellar evolution and to improve our theoretical models.

\section{References}

Kiminki, M. M., Reiter, M., \& Smith, N. 2016, MNRAS, 463, 845

Ofek, E. O., Sullivan, M., Shaviv, N. J., et al. 2014, ApJ, 789, 104

Strotjohann, N. L., Ofek, E. O., Gal-Yam, A., et al. 2015, ApJ, 811, 117 
Corsi, A., Ofek, E. O., Gal-Yam, A., et al. 2014, ApJ, 782, 42

Gal-Yam, A., Arcavi, I., Ofek, E. O., et al. 2014, Nature, 509, 471

Khazov, D., Yaron, O., Gal-Yam, A., et al. 2016, ApJ, 818, 3

Thöne, C. C., de Ugarte Postigo, A., Leloudas, G., et al. 2017, A\& A, 599, A129

Elias-Rosa, N., Pastorello, A., Benetti, S., et al. 2016, MNRAS, 463, 3894

Pastorello, A., Cappellaro, E., Inserra, C., et al. 2013, ApJ, 767, 1

Mauerhan, J. C., Smith, N., Filippenko, A., Blanchard, K. B. et al. 2013, MNRAS, 430, 1801

Duggan, G., Bellm, E., Leloudas, G., Kasliwal, M. M., \& Kulkarni, S. R. 2015, The Astronomers Telegram 7515

Elias-Rosa, N., Benetti, S., Tomasella, L., et al. 2015, The Astronomers Telegram, 7042

Smith, N., Mauerhan, J. C., \& Prieto, J. L. 2014, MNRAS, 438, 1191

Thöne, C. C., Michałowski, M. J., Leloudas, G., et al. 2009, ApJ, 698, 1307

Hillier, D. J. \& Miller, D. L. 1998, ApJ, 496, 407

Foley, R. J., Berger, E., Fox, O., et al., 2011, ApJ, 732, 32

Maeder, A. \& Meynet, G. 2000, A\& A, 361, 159

Gal-Yam, A. \& Leonard, D. C. 2009, Nature, 458, 865

Smith, N. 2014, ARA\& $A, 52,487$

Margutti, R., Milisavljevic, D., Soderberg, A. M., et al. 2014, ApJ, 780, 21

Kankare, E., Kotak, R., Pastorello, A., et al. 2015, A\& A, 581, L4

Pastorello, A., Botticella, M. T., Trundle, C., et al. 2010, MNRAS, 408, 181

\section{Discussion}

KIRSHNER: Is there any kind of relation in e.g. luminosity vs. timescale that would allow us to pick-up those pre-explosion outbursts from observations with LSST?

ТнÖNE: Interesting comment, this should certainly be given a thought for when LSST comes online.

VALENTI: Why do those events have the low Ni masses observed?

HIRSCHI: The low Ni masses are expected as these stars have massive cores that are hard to explode and hence little $\mathrm{Ni}$ is expected. 